\title{
Angiographic findings of in-stent intimal hyperplasia after stent-assisted coil embolization: are they permanent findings?
}

\author{
Young Soo Kim, MD, ${ }^{2}$ Sang Won Lee, MD, PhD, ${ }^{2}$ Jeong A Yeom, MD, ${ }^{1}$ Chang Hyo Yoon, MD, and \\ Seung Kug Baik, MD, PhD'
} Departments of ${ }^{1}$ Radiology and ${ }^{2}$ Neurosurgery, Pusan National University Yangsan Hospital, Pusan National University School of
Medicine, Yangsan, Korea

\begin{abstract}
OBJECTIVE Stent-assisted coil embolization for the treatment of intracranial aneurysms has been used widely. This study aimed to investigate the effect of stent implantation in the nonatherosclerotic parent artery with cerebral aneurysms. The authors evaluated luminal changes and the related factors following stent-assisted coil embolization.
\end{abstract}

METHODS This study included 97 patients harboring a total of 99 unruptured aneurysms of the distal internal carotid artery (ICA) who underwent single-stent implantation and more than 1 session of conventional angiography during follow-up (midterm follow-up only, $n=70$; midterm and long-term follow-up, $n=29$ ) between January 2009 and April 2014. The luminal narrowing point was measured using a local thickness map (ImageJ plug-in).

RESULTS Stent-assisted coil embolization caused dynamic luminal narrowing of approximately $82 \%$ of the parent artery diameter on average after 8 months, which was reversed to $91 \%$ after 25 months. In addition, luminal narrowing greater than $40 \%$ was noticed in $2(7 \%)$ of the 29 patients who experienced spontaneous reversion without additional management during follow-up. Most luminal narrowing changes seen were diffuse.

CONCLUSIONS Luminal narrowing after aneurysm stent-assisted coil embolization is a dynamic process and appears to be a spontaneously reversible event. Routine management of luminal narrowing may not cause adverse events that require additional treatment.

http://thejns.org/doi/abs/10.3171/2015.2.JNS142557

KEY WORDS cerebral aneurysm; coiling; endovascular; stent; stenosis; intimal hyperplasia; vascular disorders

$\mathrm{E}$ NDOVASCULAR treatment of intracranial aneurysms is a useful alternative to open clipping. A recent multicenter randomized trial demonstrated improved safety and clinical outcomes of endovascular treatment compared with neurosurgical clipping. Furthermore, an increasing trend toward endovascular treatment for aneurysm elimination is reported, and results from recent studies have shown that stent-assisted coil embolization (SACE) now accounts for about one-fourth to one-fifth of all endovascular treatments. ${ }^{1}$

However, while most SACE studies appear to be lim- ited to stent-strut apposition, parent artery conformity, and aneurysm recanalization, intimal hyperplasia after SACE in the nonatherosclerotic segment of the intracranial artery has been investigated only in a few studies, even though this treatment modality has been used clinically for more than 10 years..$^{5,-9,15}$

This retrospective study aimed to investigate the effects of stent implantation into nonatherosclerotic intracranial parent vessels with cerebral aneurysms through long-term observation, and to apply the results of this study in the clinical setting. 


\section{Methods}

The institutional review board of our hospital approved this study. We reviewed the medical records of 252 intracranial SACEs performed in 241 patients between January 2009 and April 2014.

Inclusion criteria were 1) saccular aneurysms of unruptured distal internal carotid artery (ICA); 2) single SACE; 3) nonatherosclerotic vessels without stenosis of the parent arteries; 4) aneurysms without intraoperative complications during endovascular treatment, except thrombus formation, which were successfully treated with intraarterial tirofiban (Aggrastat, Merck \& Co.); and 5) cases followed up using conventional angiography.

Although our patients presented with various conditions, we excluded patients with ruptured aneurysms and dissections, as these conditions are known to induce vasospasm and affect clinical courses. Patients who were re-treated and showed stent migration were also excluded from the study. Finally, a total of 97 patients with 99 unruptured distal ICA aneurysms were enrolled in the study. Two female patients had bilateral aneurysms of the paraclinoid ICA. Aneurysms were managed using $66 \mathrm{Neu}-$ roform EZ stents (Stryker Neurovascular), 29 Enterprise stents (Codman Neurovascular), and 4 Solitaire stents (ev3 Neurovascular). Patients underwent a single stent-assisted coil embolization and were followed up using conventional angiography. Of 99 aneurysms, 70 underwent midterm follow-up only and 29 underwent midterm and long-term follow-up. Aneurysms were located in the supraclinoid ICA $(n=73)$, ophthalmic artery $(n=19)$, posterior communicating artery $(n=6)$, and anterior choroidal artery $(n$ =1) (Table 1). The prevalence of potential risk factors for luminal narrowing after the SACE procedure, including diabetes, hypertension, preexisting vascular calcification, and hyperlipidemia, were analyzed. Follow-up angiography was performed at approximately 6 months (midterm) and approximately 24 months (long-term) after the initial SACE procedure.

\section{Endovascular Procedure}

All endovascular procedures were performed using a biplane angiography unit with 3D rotational angiography capability (Axiom Artis Zee Biplane, Siemens). All patients received clopidogrel (75 $\mathrm{mg} /$ day) and aspirin (100 $\mathrm{mg}$ /day) treatment at least 7 days before the procedure. Platelet inhibition function was measured using the multiplate analyzer system (Verum Diagnostica $\mathrm{GmbH}$ ) from June 2012 onward. An initial intravenous bolus injection of heparin (3000-5000 IU, 50 IU/kg of body weight), followed by a $1000 \mathrm{IU}$ per hour infusion was administered to all patients during the procedure. In cases of thrombus formation, 0.25-1.0 mg of intraarterial tirofiban (Aggrastat, Merck \& Co.) was administered at $0.5-1 \mu \mathrm{g} / \mathrm{sec}^{2}$ After the procedure, patients received clopidogrel and aspirin for at least 6 months. The procedure was performed in the awake state with vital sign monitoring, including continuous echocardiography, blood pressure, and heart rate.

\section{Angiographic Follow-Up}

Patients were followed up radiographically and clini-
TABLE 1. Patient demographics and aneurysm characteristics

\begin{tabular}{lc}
\hline \multicolumn{1}{c}{ Variable } & Value $^{*}$ \\
\hline No. of aneurysms & 99 \\
\hline No. of patients & 97 \\
\hline No. of sessions of follow-up angiography & \\
1 & 70 \\
2 & 29 \\
\hline Mean age in yrs ( \pm SD) & $55 \pm 9$ \\
\hline Sex distribution & \\
Male & $24(24)$ \\
Female & $75(76)$ \\
\hline Mean aneurysm size ( \pm SD) & $4.5 \pm 2.0$ \\
\hline Aneurysm location & \\
Paraclinoid ICA & 73 \\
Ophthalmic artery & 19 \\
PCoA & 6 \\
Anterior choroidal artery & 1 \\
\hline Unruptured aneurysm & $99(100)$ \\
Incidental & 68 \\
Headaches & 26 \\
CN palsy & 2 \\
Mass effect & 2 \\
TIA & 1 \\
\hline Stent & \\
Neuroform & $66(67)$ \\
Enterprise & $29(29)$ \\
Solitaire & $4(4)$ \\
\hline CN = cranial nerve; PCoA = posterior choroidal artery; TIA & transient ische- \\
mic attack. & \\
$*$ Values are presented as the number of aneurysms (\%) unless stated \\
otherwise.
\end{tabular}

cally for approximately 6 months (midterm) and 24 months (long-term) after the procedure. Angiography was performed with the working angles used during SACE. The working angles were reproduced by optimizing the orientation of bony landmarks on native images.

\section{Vessel Diameter Measurement}

Lumen changes in the stented segment were assessed using digital subtraction angiography. Full arterial phase angiograms obtained immediately after SACE and at follow-up were analyzed to reconstruct the thickness map using the local thickness plug-in for ImageJ (version 1.47) software (OptiNav Inc.) (Figs. 1 and 2). This technique allowed measurement of the entire artery thickness. ${ }^{4,6,11}$ Vessel diameter was measured at the most stenotic point of the parent artery on the first follow-up image. Each image was standardized by sequential measurements of the horizontal segment of the petrous ICA, and a ratio of each image was calculated.

\section{Data Analysis}

Descriptive statistics, including the mean and SD, was used to analyze vessel measurements. The percentage of narrowing caused by the stent was calculated using the following formula: $\mathrm{T}_{\text {follow-up }} / \mathrm{T}_{\text {initial }}$, where $\mathrm{T}$ is the thickness of the lesion/standardized thickness of the petrous ICA. 


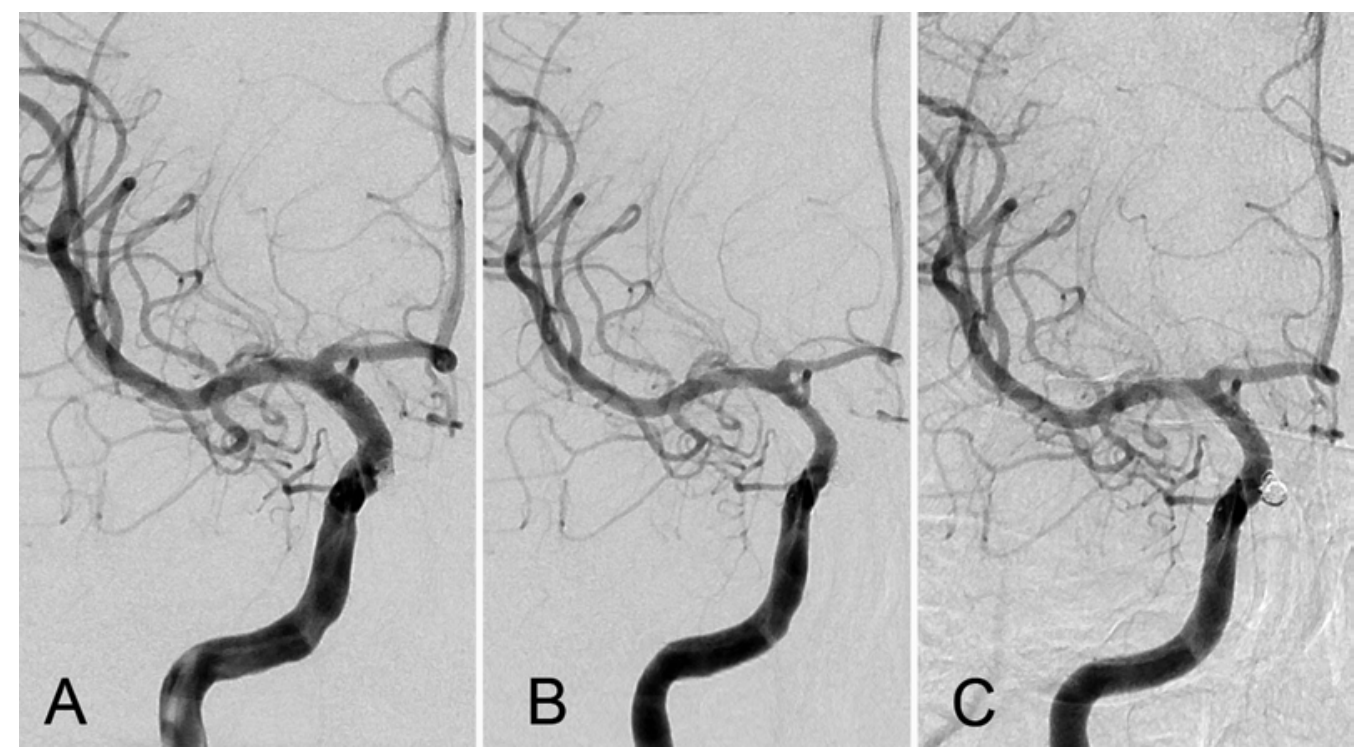

FIG. 1. Full arterial-phase angiograms obtained immediately after stent-assisted coil embolization (A) and at midterm (B) and longterm (C) follow-up assessments to reconstruct the thickness map.

We compared the mean values and $95 \%$ confidence intervals of angiograms obtained initially and at follow-up, and the change over time in every group was examined using the Bonferroni method. The angiographic pattern of luminal narrowing was categorized as focal or diffuse with or without a focal lesion (coil distal, articulation, or diffuse in-stent). A paired Student t-test was used to evaluate the statistical significance of diameter change, and an intraclass correlation coefficient was used to determine the level of interobserver reliability for the classification. All statistical analyses were performed using SPSS statistical software (version 18.0, SPSS Inc.).

\section{Results}

Midterm and long-term follow-up angiography was per- formed within a mean of 7.9 months (range 6-13 months) and a mean of 24.5 months (range 17-36 months) after the procedure, respectively. Compared with the mean diameter of the in-stent vessel measured immediately after the procedure $(100 \%)$, the mean diameter decreased to $82 \%$ as shown in the midterm follow-up, whereas it had increased to $91 \%$ by the time of the long-term follow-up assessment (Fig. 3); no differences among the types of the stent were observed. Furthermore, 2 of the 29 patients (7\%) who underwent midterm and long-term follow-up assessment showed luminal narrowing above $40 \%$ at the time of the midterm follow-up but a significant recovery of the luminal narrowing up to $91 \%$ of the original diameter was observed in the long-term follow-up angiography. A luminal change greater than $10 \%$ was seen in 28 of 29 patients with longterm follow-up, and these changes could be categorized
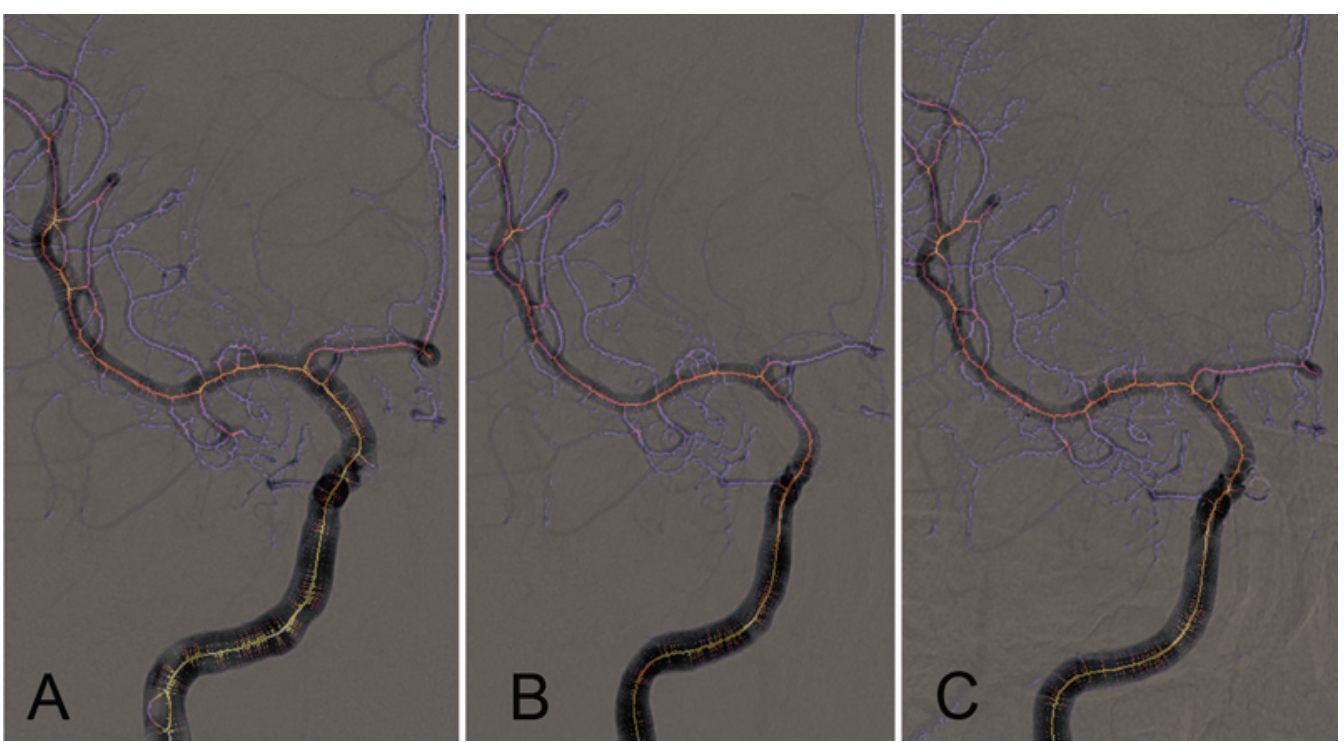

FIG. 2. ImageJ local thickness plug-in. Image of a paraclinoid ICA aneurysm with a wide neck (A), at the midterm follow-up assessment (B), and at the long-term follow-up assessment (C). Figure is available in color online only. 


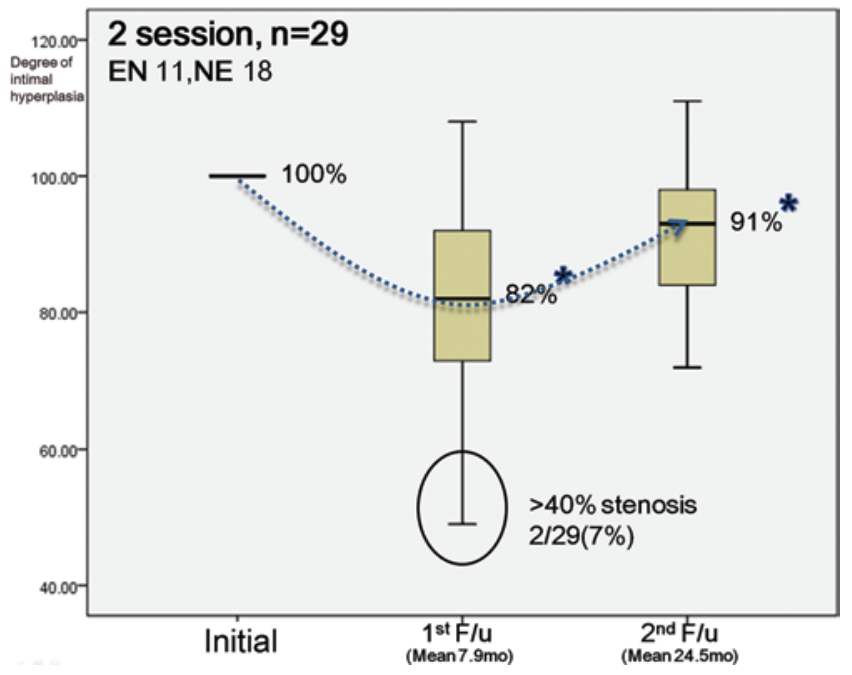

FIG. 3. The mean diameter of the in-stent vessel was smaller (82\%) at the time of the midterm follow-up than that measured immediately after stent-assisted coil embolization (100\%); by the time of the long-term follow-up assessment, it had increased to $91 \%$. The severe luminal narrowing seen in 2 patients at the midterm follow-up had recovered up to $90 \%$ by the time of the long-term follow-up assessment. ${ }^{*} p<0.05$. EN = Enterprise stent; $F / u=$ follow-up; NE = Neuroform EZ stent. Figure is available in color online only.

morphologically. Most luminal narrowing was categorized as diffuse and diffuse with focal lesion type $(\mathrm{p}<0.05)$ (Fig. 4). The lesions were assessed and categorized by 2 neurointerventionists, with good agreement overall (Fleiss' generalized kappa in a subcohort of 28 cases was 0.72 ) regarding the severity of luminal narrowing. All patients showing luminal narrowing underwent routine follow-up angiography and received treatment with antiplatelet drugs, with no additional treatment being required.

Digital subtraction angiography was performed in 99 aneurysms to evaluate luminal narrowing. The postop- erative time was divided into 5 time points as initial, 6-9, 10-15, 16-24, and over 24 months. Diameters measured at each time period were compared with those measured immediately after SACE $(100 \%)$, and a similar reversible narrowing was observed ( $p<0.001$, Bonferroni method). The average luminal narrowing at the 6- to 9-month period was $82 \%$. As a result of the continual improvement shown over the long-term follow-up period, the narrowing rose to $92 \%$ (Fig. 5).

Results from analyses of potential risk factors for luminal narrowing after SACE, including diabetes, hypertension, preexisting vascular calcification, and hyperlipidemia, did not reach statistical significance.

\section{Discussion}

SACE has been used as the treatment for $20 \%-25 \%$ of aneurysms. However, this procedure is being increasingly used because of its larger aneurysm neck coverage, feasibility, and long-term stability. ${ }^{17}$ SACE appears to have different effects with respect to luminal narrowing of the normal parent artery in patients who undergo standard coiling. During the first weeks to months of the midterm phase that follows the intervention, smooth muscle cells migrate from the tunica media to the tunica intima and undergo proliferation, which leads to thickening of the intima. Therefore, intimal hyperplasia is a main concern in the midterm phase of luminal narrowing. ${ }^{3,10}$ The longterm phase continues for months to years, and during this period the cellular infiltrates gradually resolve and the endothelium becomes fully mature. Therefore, mature intimal hyperplasia can be grossly indistinguishable from that of an adjacent nonstented artery. ${ }^{13}$

In-stent stenosis is a relatively well-known complication of coronary artery disease treatment, with an incidence of $10 \%-50 \% .^{13}$ It is generally thought that in-stent stenosis following stenting of a stenotic coronary artery

\section{Type I -focal lesion (4\%)

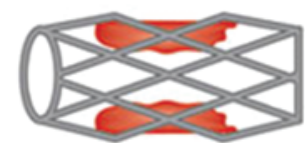 \\ $1 / 28$ \\ Type I: focal}

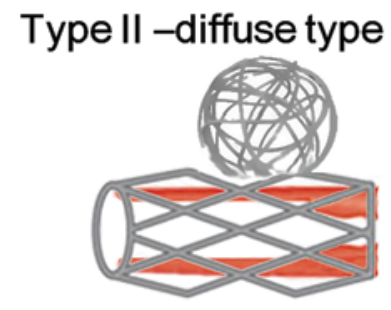

Type IIA: coil distal

$6 / 28$

(21\%)

with/without focal lesion (96\%)

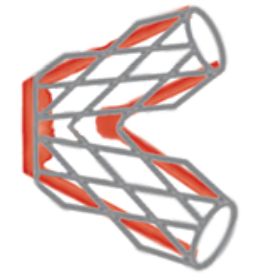

Type IIB: articulation

$6 / 28$

(21\%)

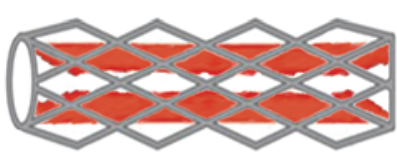

Type IIC: diffuse in-stent

$15 / 28$

$(54 \%)$

FIG. 4. Types of intimal hyperplasia. In all, 28 of 29 patients (96\%) showed that a luminal change of more than $10 \%$ could be analyzed and were categorized as intimal hyperplasia types. Most intimal hyperplasia changes were of the diffuse and diffuse with focal lesion type $(p<0.05)$. Figure is available in color online only. 


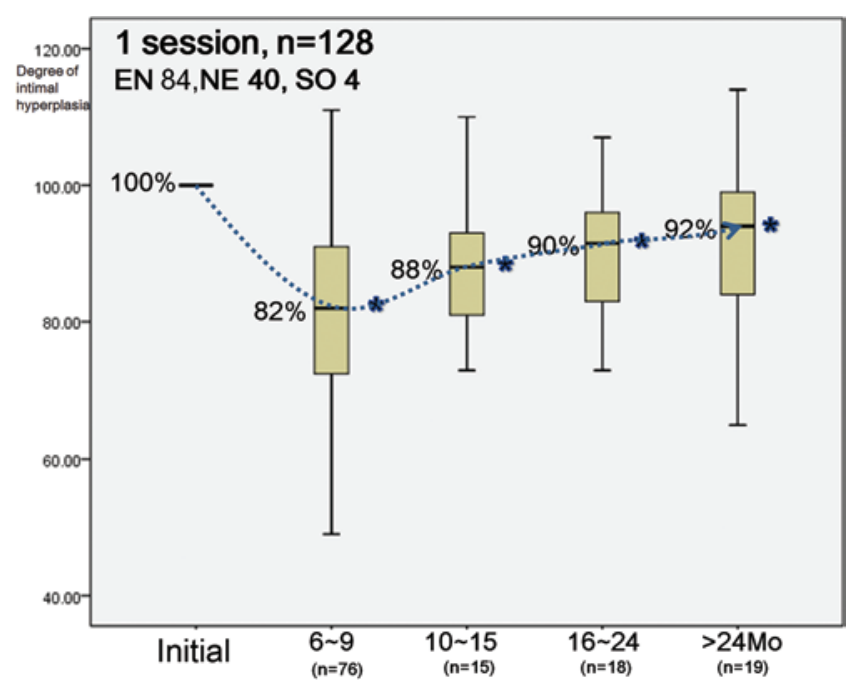

FIG. 5. Patients who underwent more than 1 angiography follow-up session had 99 aneurysms. The postoperative time was divided into 5 time points, 6-9, 10-15, 16-24, and more than 24 months. Diameters measured at each time period were compared with those measured immediately after stent-assisted coil embolization (100\%). Although a decrease was observed at the 6 - to 9 -month period $(82 \%)$, by the longterm follow-up, the mean diameter had increased $(92 \%)$. ${ }^{\star} p<0.05$. SO = Solitaire stent. Figure is available in color online only.

results primarily from neointimal hyperplasia caused by localized reaction to an arterial wall injury generated during inflation of balloon-expandable stents, which leads to the progression of primary atherosclerotic disease. ${ }^{14}$ However, the mechanism may not explain the intimal thickening provoked by SACE because the stent is placed in a normal parent artery.

This study aimed to investigate the effect of stent implantation into nonatherosclerotic intracranial parent vessels with cerebral aneurysms. Our results suggest that about $20 \%$ of luminal narrowing occurs in the majority of patients during the midterm follow-up period (mean 8 months). A postmortem study of ICA aneurysms treated with the Neuroform stent reportedly showed that the stent itself abuts the internal elastic lamina, with moderate intimal hyperplasia observed 4 months after single-stent deployment..$^{12}$ Moreover, results from a study by Schatz et al. ${ }^{16}$ demonstrated that the stent is initially covered by a thin layer of thrombus and then replaced by a proliferated neointimal muscle layer that reaches maximal thickness by 2 months, whereas the neointima gradually becomes thin and more sclerotic and less cellular by 8 months. In our study, intimal hyperplasia occurred within 8 months and resolved spontaneously within 24 months, leading us to estimate that intimal hyperplasia may have reached its peak between 2 and 8 months.

Recently, Gao et al. 7 reported that although Enterprise stenting induces dynamic and reversible age-dependent stenosis in the cerebral arteries and that stenosis in the distal part of the stent is most severe after 4-6 months, patients recover in a time-dependent manner after 12-24 months. In our study, no differences were found among stent type. Furthermore, the mean diameter of the parent artery decreased to $82 \%$ of the original diameter after 8 months, but increased to $91 \%$ after 24 months ( $<0.05)$.
In addition, our results indicated that by the time of the midterm follow-up assessment (mean 8 months), significant luminal narrowing had occurred in some patients (7\% of our patients), but this was a temporary event that resolved within 2 years.

The angiographic pattern of intimal hyperplasia is generally categorized into 2 types, i.e., diffuse or diffuse with focal lesion type; most luminal narrowing in our study was categorized as diffuse and diffuse with focal lesion type. A number of possible factors that lead to luminal narrowing can be considered. First, in addition to diffuse reactions caused by the stent, reactions caused by the coil lead to further luminal narrowing, an event described to be caused by implanted coils. ${ }^{15}$ Second, endothelial stimulation caused by chronic radial force may occur when a large mismatch between the diameter of the parent artery and the size of the stent exists. Third, the foreign body reactions of stent's distal marker and intimal injury caused by the distal part of the stent close to the transitional zone may also play a role.

Moreover, disruption of the internal elastic lamina was found to cause neointimal hyperplasia in an animal study ${ }^{16}$ Although other reasons, such as cerebral vasospasm, inflammation caused by foreign bodies or other various causes, should also be considered, patients with ruptured aneurysms and dissections were excluded from our study because of the potential for these conditions to induce vasospasm and affect the different clinical courses, causes of intimal hyperplasia appear to be multifactorial and include coil and stent factors.

Even though routine treatment of patients with more than $40 \%$ of luminal narrowing did not cause adverse events and thus did not require additional treatment, these patients still need to undergo follow-up. Noninvasive CT and MR angiography are more frequently used than catheter angiography as a follow-up modality, although there are variations in the detection rate of small luminal changes between modalities. Therefore, if follow-up is required for a patient, the same noninvasive modality and machine should be performed as was used previously.

If significant luminal narrowing is shown on angiography 6 months after SACE, antiplatelet medications should be administered for an additional 6-month period based on platelet resistance test results. However, medications could be interrupted in patients who show recovery of the luminal narrowing detected through follow-up.

Our study has some limitations, i.e., the retrospective nature of the study and the small sample size.

\section{Conclusions}

In SACE, luminal narrowing is a dynamic process involving changes that may reverse spontaneously. Most luminal narrowing changes are diffuse and diffuse with focal lesion type, and intimal hyperplasia could be the main cause of luminal narrowing. Routine management of patients with intimal hyperplasia does not induce adverse events that require additional treatment.

\section{References}

1. Alshekhlee A, Mehta S, Edgell RC, Vora N, Feen E, Mohammadi A, et al: Hospital mortality and complications of elec- 
tively clipped or coiled unruptured intracranial aneurysm. Stroke 41:1471-1476, 2010

2. Baik SK, Oh SJ, Park KP, Lee JH: Intra-arterial tirofiban infusion for partial recanalization with stagnant flow in hyperacute cerebral ischemic stroke. Interv Neuroradiol 17:442-451, 2011

3. Chalouhi N, Drueding R, Starke RM, Jabbour P, Dumont AS, Gonzalez LF, et al: In-stent stenosis after stent-assisted coiling: incidence, predictors and clinical outcomes of 435 cases. Neurosurgery 72:390-396, 2013

4. Dougherty RP, Kunzelmann KH: Computing local thickness of 3D structures with ImageJ. Microsc Microanal 13:16781679,2007

5. Ebrahimi N, Claus B, Lee CY, Biondi A, Benndorf G: Stent conformity in curved vascular models with simulated aneurysm necks using flat-panel CT: an in vitro study. AJNR Am J Neuroradiol 28:823-829, 2007

6. Fischer MJ, Uchida S, Messlinger K: Measurement of meningeal blood vessel diameter in vivo with a plug-in for ImageJ. Microvasc Res 80:258-266, 2010

7. Gao B, Baharoglu MI, Cohen AD, Malek AM: Stent-assisted coiling of intracranial bifurcation aneurysms leads to immediate and delayed intracranial vascular angle remodeling. AJNR Am J Neuroradiol 33:649-654, 2012

8. Heller RS, Malek AM: Parent vessel size and curvature strongly influence risk of incomplete stent apposition in enterprise intracranial aneurysm stent coiling. AJNR Am J Neuroradiol 32:1714-1720, 2011

9. King RM, Chueh JY, van der Bom IM, Silva CF, Carniato SL, Spilberg G, et al: The effect of intracranial stent implantation on the curvature of the cerebrovasculature. AJNR Am J Neuroradiol 33:1657-1662, 2012

10. Lee DH, Arat A, Morsi H, Diaz O, Jou LD, Mawad ME: Intimal thickening after placement of a neuroform stent. its incidence and relation to angiographic follow-up results of aneurysm embolization. Interv Neuroradiol 13:239-246, 2007

11. Liuhanen S, Sallisalmi M, Pettilä V, Oksala N, Tenhunen J: Indirect measurement of the vascular endothelial glycocalyx layer thickness in human submucosal capillaries with a plug-in for ImageJ. Comput Methods Programs Biomed 110:38-47, 2013

12. Lopes D, Sani S: Histological postmortem study of an inter- nal carotid artery aneurysm treated with the Neuroform stent. Neurosurgery 56:E416, 2005

13. Lowe HC, Oesterle SN, Khachigian LM: Coronary in-stent restenosis: current status and future strategies. J Am Coll Cardiol 39:183-193, 2002

14. O'Connell BM, McGloughlin TM, Walsh MT: Factors that affect mass transport from drug eluting stents into the artery wall. Biomed Eng Online 9:15, 2010

15. Piotin M, Blanc R, Spelle L, Mounayer C, Piantino R, Schmidt PJ, et al: Stent-assisted coiling of intracranial aneurysms: clinical and angiographic results in 216 consecutive aneurysms. Stroke 41:110-115, 2010

16. Schatz RA, Palmaz JC, Tio FO, Garcia F, Garcia O, Reuter SR: Balloon-expandable intracoronary stents in the adult dog. Circulation 76:450-457, 1987

17. White PM, Lewis SC, Nahser H, Sellar RJ, Goddard T, Gholkar A: HydroCoil Endovascular Aneurysm Occlusion and Packing Study (HELPS trial): procedural safety and operator-assessed efficacy results. AJNR Am J Neuroradiol 29:217-223, 2008

\section{Disclosure}

The authors report no conflict of interest concerning the materials or methods used in this study or the findings specified in this paper.

\section{Author Contributions}

Conception and design: Baik. Acquisition of data: Kim. Analysis and interpretation of data: Kim. Drafting the article: Kim. Critically revising the article: Baik, Kim. Reviewed submitted version of manuscript: Kim, Lee, Yeom, Yoon. Approved the final version of the manuscript on behalf of all authors: Baik. Statistical analysis: Kim. Administrative/technical/material support: Lee. Study supervision: Baik.

\section{Correspondence}

Seung Kug Baik, Department of Radiology, Pusan National University Yangsan Hospital, Pusan National University School of Medicine, 20, Geumo-ro, Mulgeum-eup, Yangsan-si, Gyeongsangnam-do 626-770, Korea. email: skbaik9@gmail.com. 